\title{
Enhanced light emission from dye doped polymer layers using surface plasmons
}

\author{
Terrell D. Neal, ${ }^{\text {a) }}$ Koichi Okamoto, and Axel Scherer \\ Department of Electrical Engineering, California Institute of Technology, Pasadena, CA 91125 \\ terrell@caltech.edu
}

\begin{abstract}
We have experimentally verified that the emission of visible light from dye doped polymers can be enhanced with the use of surface plasmon coupling. We have observed an eleven-fold enhancement of light emission.

(C) 2005 Optical Society of America

OCIS codes: (240.6680) Surface plasmons; (250.3680) Light-emitting polymers
\end{abstract}

The large optical fields provided by surface plasmons have in the past been used to significantly increase the fluorescence and luminescence intensity of dye molecules for applications in biological and biochemical sensing. Molecules can be detected on the surface of metal films or in close proximity to colloidal metal particles by measuring increases in the resonant surface plasmon coupled emission [1]. Recently, it has also been shown that plasmon resonances provided by thin metal layers and metal gratings can be designed to increase the efficiency of solid state semiconductor light sources. In such structures, the surface plasmon resonance frequency is tuned to the semiconductor quantum well emission, and the light emission intensity can be increased by over an order of magnitude as nonradiative recombination paths are bypassed through coupling of recombination energy into surface plasmon modes. It is therefore not surprising that similar energy transfer into surface plasmons can also be exploited in the design of more efficient organic light emitters.

Polymers, appropriately doped with dye molecules, emitting in the visible spectrum provide stable sources of light for displays and illumination sources at a significantly lower cost than semiconductors. Organic light emitting diodes [2,3] (OLEDs) may indeed evolve as the most inexpensive alternatives to fluorescent light sources for large area solid state lighting. Thus, it is of both commercial and scientific interest to improve the internal quantum efficiencies of the polymer dyes within such light emitters, as well as to increase the light extraction efficiencies from such organic films. Indeed, the effect of surface plasmons on the light emission from polymer layers has been recently been investigated by several groups [4-6]. Here, we focus on enhancing the light emission efficiency from organic thin films by using the surface plasmon resonance.

The experimental setup used to measure our samples is shown in our earlier work [7]. To ensure that only a small amount of the pump light is reflected into the detector, the pump source was introduced at a large incidence angle while the detector was located perpendicular to the sample. We compare the influence of gold and silver thin films on the light emission of a commonly used laser dye doped in a polymer by using this measurement system. Of course, the light emitting material must be within a few hundred nanometers of the metal surface to benefit from surface plasmon enhancements. For semiconductor quantum wells, this is normally accomplished by growing a very thin spacer separating the quantum well from the metal surface. For polymer layers, it is much simpler to prepare measurable samples, as it is only necessary be ensure that the pump light is not totally absorbed by the dye layer.

Figure 1 shows a typical measurement obtained when comparing the spectra of a region with Coumarin 460 doped PMMA spun onto gold with that of the polymer on the bare quartz substrate, where the Coumarin $460 \mathrm{PL}$ intensisty is normalized to 1 . While the gold assisted in reflecting the pump laser, the surface plasmons did not seem to couple to the emission wavelength of Coumarin 460 to offer any measurable enhancement. However, we do observe an 11 fold enhancement of the emission light from the Coumarin 460 doped PMMA on silver due the coupling of the surface plasmons generated on the silver film as the plasmon resonance frequency closely matches the emission frequency of the dye. Indeed, the dielectric constants for silver match well with the emission wavelength of Coumarin 460. While reflection can be used to account for some of the increased brightness, only the SP coupling can explain the enhancement measured. 


\section{CTuE4.pdf}

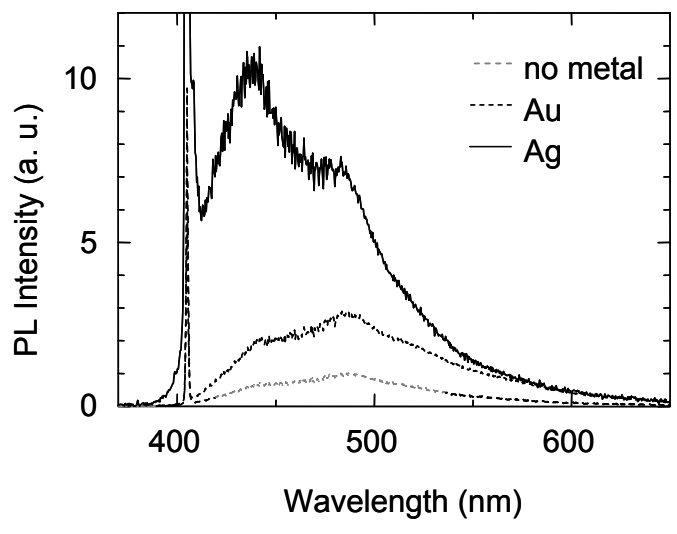

Fig. 1. PL spectra.

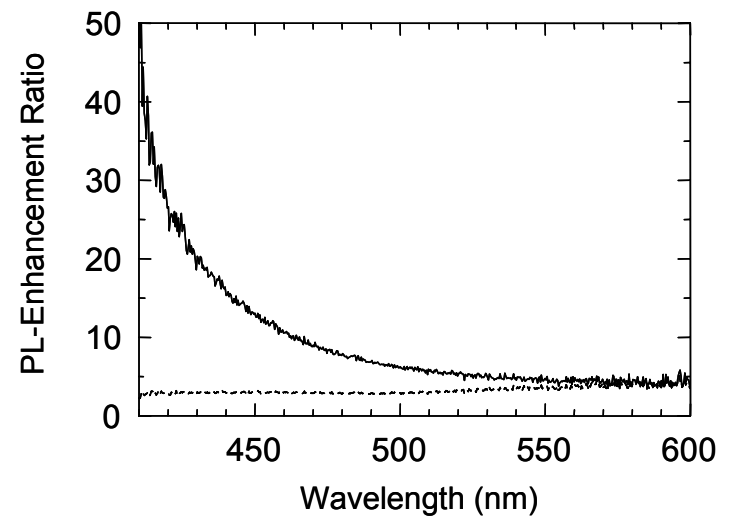

Fig. 2. PL Enhancement ratios.

Enhancement ratios illustrated in Fig. 2 further support the notion of SP coupling between the silver and polymer interface. Up to 50 times enhancements are observed along the broad emission spectrum of the dye doped polymer whereas at the peak emission wavelength the photoluminescence enhancement ratio is 11 times. We observed an increase of photoluminescence enhancement ratio with shorter wavelengths for the silver film. However, the PL enhancement ratio is not strongly dependent on the change in wavelength for the gold film.

The dramatic PL enhancement of samples with Ag can be attributed to the strong interaction between the excited dye molecules and SPs. Electron-excited energy of molecules can couple to the electron vibration energy of SP. Then, the molecular relaxation processes may produce a SP instead of a photon, and this new path of the relaxation increases the spontaneous recombination rate. If the metal surface was perfectly flat, it would be difficult to extract light emission from the SP, since it is a non-propagating evanescent wave. However, roughness and imperfections in evaporated metal can scatter SPs as light. The coupling rate between the excited molecules and SPs is expected to be much faster than the nonradiative relaxation processes as a result of the large electromagnetic fields introduced by the large density of states.

We have observed that the emission of dye doped polymers can be enhanced by using surface plasmon coupling to thin metal layers. This study serves as a foundation for the geometric tuning of the surface plasmon resonance and enhancement of the emission of dye polymers using surface plasmons with patterned metal samples. If tuned properly, such lithographically structured layers should provide even higher enhancement values for the dye emission intensity. As the metallic surface can be used as an electrical contact, as a metallic grating for enhanced light extraction, and as a mirror for the definition of ultra-small optical cavities to further increase the spontaneous emission rates, we expect that many organic light-emitting diodes could benefit from careful design and choice of metallization. Ultimately, lithographic tuning of the peak emission wavelength and optimization of the polymer layer stack can also result in efficient white light source OLEDs.

[1] Malicka, J., et al., "Use of surface plasmon-coupled emission to measure DNA hybridization," J. Biomol. Screening, 9, 208-215 (2004)

[2] Jiang, X.Z., et al., "Organic light-emitting diodes using an in situ thermally polymerized hole transporting layer," Appl. Phys. Lett., 76, 2985-2987 (2000).

[3] Carlson, B., et al., "Divalent osmium complexes: Synthesis, characterization, strong red phosphorescence, and electrophosphorescence," J. Am. Chem. Soc., 124, 14162-14172 (2002).

[4] Hobson, P.A., et al., "Surface plasmon mediated emission from organic light-emitting diodes," Advanced Materials, 14, 1393-1396 (2002).

[5] Andrew, P. and W.L. Barnes, "Energy transfer across a metal film mediated by surface plasmon polaritons," Science, 306, 1002 1005 (2004).

[6] Alencar, M., A.S.L. Gomes, and C.B. de Araujo, "Directional laserlike emission from a dye-doped polymer containing rutile nanoparticles," J. Opt. Soc. Am. B-Optical Physics, 20, 564-567 (2003).

[7] Neal, T.D., Okamoto, K., Scherer, A., "Surface plasmon enhanced emission from dye doped polymer layers," Optics Express 13, $5522-5527(2005)$ 